Rev. SINAPSIS, Vol. 5, No 2, Diciembre 2014

ISSN $1390-7832$

\title{
La pizarra didáctica cartesiana para el trabajo en el laboratorio de matemáticas
}

\section{La pizarra cartesiana y las matemáticas}

Julio César Silva Ruiz. Mg.Sc. ${ }^{(1)}$

Roberth Zambrano Santos. Ph.D. ${ }^{(2)}$

(1) Unidad Educativa ITSUP. Universidad San Gregorio de Portoviejo, Ecuador

${ }^{(2)}$ Instituto Tecnológico Superior Portoviejo. Universidad Estatal del Sur de Manabí, Ecuador

Contacto: $\underline{\text { srjcjulio@yahoo.es }}$

\section{Receptado: 15/07/2014 Aceptado: 22/9/2014}

Los rápidos cambios en la ciencia y la tecnología hacen que una mayor atención a las materias que se imparten en las escuelas, incluyendo las matemáticas, lo que constituye una de las claves en el proceso. El objetivo de este trabajo fue encontrar nuevos mecanismos para estimular la enseñanza de las matemáticas, que debe ser enfocada en el desarrollo de competencias con los criterios de rendimiento necesarios para que el estudiante sea capaz de resolver los problemas de todos los días, mientras que el pensamiento lógico y crítico impuesto por el mundo moderno. Se llevó a cabo en la Unidad Educativa ITSUP en Ecuador. Se propone el uso de la pizarra cartesiana en la enseñanza para fortalecer este proceso con los movimientos de arte, donde se manipulan y lograr con el objetivo de clase de objetos. Para esta propuesta, se utilizó una metodología que facilita la información que se encuentra en un tablero con agujeros en un plano con el apoyo de dos reglas, una horizontal y una vertical, lo que indica la representación de un nivel secundario, que se sintetiza en el plano cartesiano. Se concluyó que las operaciones que requieren el uso de avión Descartes, sobre la cual el gráfico lineal o funciones cuadráticas se proporciona. Mayor dinamismo en la clase se logra y el deseo espontáneo de conocimiento de los estudiantes.

Palabras clave: Metodología matemática, enseñanza matemática, aprendizaje de matemática, herramienta matemática

The Cartesian blackboard teaching to work in the math lab 


\begin{abstract}
The rapid changes in science and technology makes greater attention to the subjects taught in schools, including mathematics, which constitutes one of the key in the process. The objective of this work was to find new mechanisms for stimulating the teaching of mathematics, which should be focused on developing skills with performance criteria necessary for the student to be able to solve everyday problems, while the logical and critical thinking imposed by the modern world. It was carried out in Education Unit ITSUP in Ecuador. It proposes the use of Cartesian blackboard in teaching to strengthen this process with the art movements, where objects are manipulated and achieved with the class objective. For this proposal, it was used a methodology that facilitates the information that lies on a board with holes in one plane with the support of two rules, a horizontal and a vertical one, indicating the representation of a secondary level, which is synthesized in the Cartesian plane. It was concluded that operations requiring the use of Descartes plane, on which to linear graph or quadratic functions is provided. Greater dynamism in class is achieved and the spontaneous desire of knowledge of students is developed.
\end{abstract}

Keywords: mathematical methodology, mathematics teaching, learning, math, mathematical tool

\title{
Introducción
}

El principal problema que se trata de solucionar con este trabajo es la poca afinidad que tienen los estudiantes con el aprendizaje de matemática, principalmente cuando se trata de graficar funciones y encontrar soluciones que no le faciliten su observación.

Este trabajo es producto de un proyecto de investigación que recoge el esfuerzo académico en las aulas de clases, ante las miradas frías de los estudiantes, cuando se trataba de dar solución a los problemas de programación lineal que incluyen graficas de ecuaciones y restricciones, en el campo de la sección de las cónicas: parábola, elipse, hipérbola, incluyendo a la circunferencia, especialmente cuando las traslaciones de ejes se presentan, y la necesidad de identificar a todos los elementos de dichas figuras. 
Rev. SINAPSIS, Vol. 5, No 2, Diciembre 2014

ISSN 1390 - 7832

La pizarra didáctica cartesiana es una especie del ábaco que fue construido hace más de 2000 años de esta era en Mesopotamia, para facilitar el uso de las operaciones básicas fundamentales como son: suma, resta y multiplicación, siendo este instrumento el que inspiro a otros hombres de ciencia a elaborar la calculadora y posteriormente la computadora.

La pizarra didáctica cartesiana puede ser utilizada dentro de las clases de matemáticas o en el laboratorio de matemáticas, siendo necesario que toda Institución Educativa la posea, a la misma se le pueden incluir elementos complementarios en cada problema para ser identificados, manipulados y además dotar indirectamente al estudiante de la posibilidad de poder inventar nuevos ejercicios con menor y mayor grado de dificultad a medida que sus conocimientos se van incrementando.

Cabe indicar que la presentación de la pizarra didáctica cartesiana en el laboratorio o en la clase de matemáticas puede reemplazar a las Tics que se utilizan para recrear un ejercicio, o utilizar la computadora para apoderarse de un sistema que está en Internet y hacer uso del mismo, brindando la pizarra didáctica cartesiana la fortaleza de poderla manipular y ser dinámicos al efectuar el desarrollo de un ejercicio o problema.

Existen un sinnúmero de formas de poder graficar, resolver ecuaciones, inecuaciones, funciones lineales o funciones cuadráticas, y diferentes metodologías utilizadas por los profesores de matemáticas para poder llegar con el conocimiento al estudiante, pero esta nueva forma de poder alcanzar el objetivo del tema o de la unidad respectiva es novedosa y da luces a los profesores que la utilizan al incluir nuevos temas que antes no la aplicaban.

Una pizarra es una superficie donde se escribe textos, figuras, o se grafica con tizas o con marcadores, lo que históricamente está evolucionando hasta estos días, si se le agrega el termino didáctica, “...literalmente Didáctica en su doble raíz docere; enseñar y discere aprender, vocablos que significan realizar actividades de enseñar y aprender, y que reclaman la interacción de los agentes que la realizan...” (Medina \& Francisco, Didactica General, 2009). Desde una visión activo-participativa, el docente es aquel que enseña pero a la vez es el que más aprende en este proceso de mejora continua de la tarea de co-aprender con los colegas y los estudiantes. La segunda acepción hace mención al que aprende, capaz de aprovechar una enseñanza de calidad 
Rev. SINAPSIS, Vol. 5, No 2, Diciembre 2014

para comprenderse a sí mismo y dar respuesta a los continuos desafíos del mundo en permanente cambio.

El docente podrá crear problemas para que el estudiante tenga un desafío y aprenda de estos, puede también generar un ambiente a través de herramientas como (películas, cine, etc.) pero siempre deberá dejar claro que existe un objetivo y enmarcarlo para que el estudiante se concentre en obtener la información que le permitirá cumplir con este, algunas veces esos procesos de aprendizaje no culminan de manera efectiva porque el estudiante puede tener impedimentos o dificultades para aprender. (Carvajal, 2000).

Los docentes en matemáticas deben cumplir con estándares para fomentar el razonamiento lógico matemático, la lectura y escritura matemática, las estrategias a seguir para conseguir que los estudiantes desarrollen sus propias habilidades y creatividad, aunque es cierto que la tecnología evoluciona con gran rapidez quedando en la mayoría de los casos obsoleta cuando aún se está aprendiendo a manejarla, la aparición de la pantalla digital en el aula trae un amplio abanico de oportunidades para la enseñanza; sin embargo, la comparación entre la pizarra didáctica que se propone en esta investigación y una pizarra digital u otras herramientas tecnológicas de la información y la comunicación, el interés que se logra en los estudiantes a través de la "pizarra didáctica cartesiana" es que esta brinda la posibilidad de conseguir una intersección entre la interactividad técnica y pedagógica.

El docente debe comprender la interactividad, y ello no significa utilizar simplemente la pantalla digital en el aula y que esto le resulte fácil, sino debe promover el intercambio de docentes y estudiantes a través del diálogo y el compartir actividades didácticas en conjunto. Por ello es importante que los docentes elijan estrategias y métodos de enseñanza y diseñen diferentes actividades pedagógicas apropiadas.

Bajo el contexto analizado en el párrafo anterior, se define entonces a la pizarra didáctica cartesiana como el lugar físico donde el docente y los estudiantes se encontraran para resolver por medio de herramientas y materiales complementarios aplicando los cálculos respectivos, ejercicios y problemas. Existen útiles pizarras que son aprovechadas en los laboratorios de matemáticas de las instituciones educativas, e históricamente han ido evolucionando de acuerdo al nivel de vida alcanzado, posiblemente la pizarra digital sea el mejor medio que se tenga y ésta 
Rev. SINAPSIS, Vol. 5, No 2, Diciembre 2014

consiste en un ordenador multimedia conectado a Internet y un video proyector cuyas imágenes se verán reflejadas en una pizarra blanca, en la que el docente tiene múltiples opciones, ya sea el de crear su propia información, importarla de Internet, o trabajar con materiales de videos. (Pere \& Dvoskin, 2010), sin embargo este tipo de pizarra no logra la interacción creativa entre el docente y los estudiantes, no permite la manipulación física de herramientas de trabajo que desarrollan habilidades y destrezas matemáticas muy importantes.

La pizarra didáctica cartesiana es la herramienta que toda institución educativa requiere especialmente en estos tiempos en que la tecnología se ha impuesto en la formación del estudiante, los centros educativos modernos tratan de demostrar los avances tecnológicos de sus enseñanzas y buscan en los equipos de última generación el instrumento adecuado para mejorar la calidad, disponiendo en sus laboratorios no solo de matemáticas, sino también de física, ciencias naturales, química, entre otros, con un considerable número de herramientas didácticas.

Los profesores de matemáticas están frente a nuevos retos, al tratar de mejorar el ambiente educativo para obtener mejores resultados académicos, el proceso de aprendizaje se nutrirá de otras actividades en el aula, y por lo tanto se estará estimulando el desarrollo del conocimiento desde otra perspectiva innovadora con la utilización de herramientas como lo es la pizarra didáctica cartesiana, aportando al cumplimiento del modelo de educación nacional, el cual está orientado a elevar la calidad educativa a fin de que los individuos puedan alcanzar los estándares más altos de aprendizaje, desarrollándose de manera integral, utilizando todas sus capacidades y potencial como personas y ciudadanos productivos, responsables, solidarios para que contribuyan al desarrollo de la sociedad. (ARSA, 2012).

Ante la posible polémica que se pueda presentar entre los defensores de la pizarra digital y la pizarra cartesiana que se está proponiendo en esta investigación, se hace necesario precisar que las pizarras didácticas, sean estas manuales o digitales, deben cumplir con un proceso pedagógico de evaluación para poder concluir y sacar juicios que indiquen resultados de impacto y permitan determinar que metodología fue mejor, proceso en el cual todos los docentes sacarán sus propias conclusiones.

Las actividades científicas que se desarrollaron en esta investigación fueron: Identificar la metodología para resolver en la pizarra didáctica cartesiana ejercicios de programación lineal, 
Rev. SINAPSIS, Vol. 5, No 2, Diciembre 2014

graficar funciones lineales o cuadráticas, crear ejercicios sobre las cónicas, reconocer los elementos de las cónicas; además de, fundamentar pedagógica y didácticamente los procesos metodológicos a seguir para alcanzar los objetivos de la asignatura de matemática.

\section{Desarrollo}

En cuanto a los aspectos técnicos de la pizarra didáctica cartesiana, esta consta de una superficie plana de 2,5m de largo y 1,5 m de ancho, toda la superficie esta agujereada a una distancia de 5,5 $\mathrm{cm}$, posee dos reglas: una vertical y otra horizontal de aluminio que le permite trasladarse a lo largo y ancho de la pizarra. En la parte central está definido el plano cartesiano principal que lo delimita en cuatro cuadrantes.

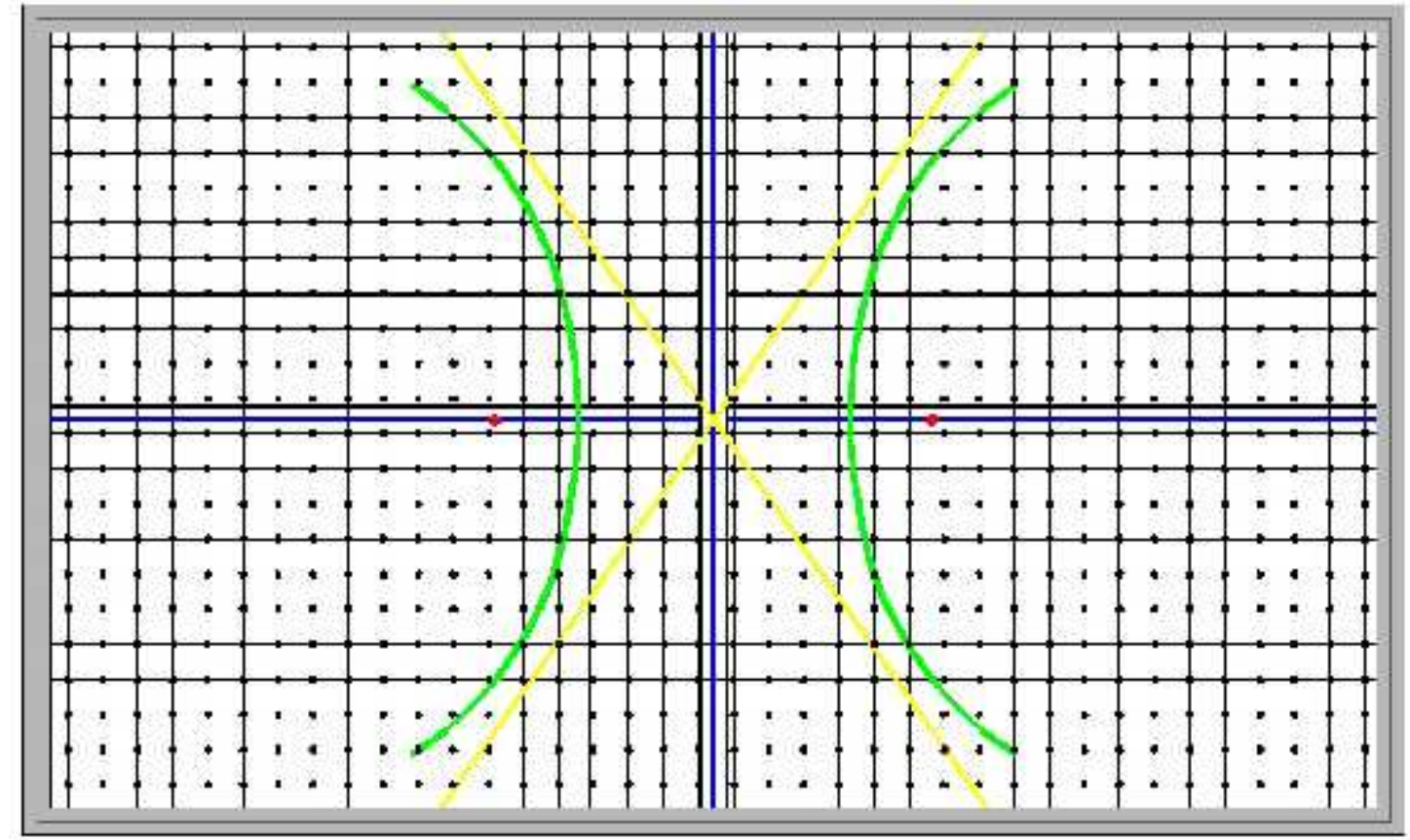

Para realizar las gráficas se tienen elementos complementarios como son: círculos, elipses, parábolas e hipérbole las mismas que fueron elaboradas técnicamente con las dimensiones de la pizarra para que la relación de correspondencia sea valedera y puedan las gráficas ser exactas, para que las figuras pre elaboradas se mantengan fijas se hizo unos soportes pequeños que entran en los agujeros de la pizarra.

Las asíntotas de la hipérbole se las utiliza en programación lineal para indicar los límites de las restricciones, también sirven para graficar funciones lineales y funciones cuadráticas. Al realizar 
Rev. SINAPSIS, Vol. 5, No 2, Diciembre 2014

ISSN 1390 - 7832

el análisis de las funciones en su dominio y codominio se los puede identificar de una mejor forma y poderlos visualizar ya que las curvas se pueden maniobrar manualmente.

Otra utilidad también es la explicación del teorema de Pitágoras colocando los triángulos rectángulos y sus cuadrados en los respectivos lados, los mismos que son observados y manipulados por los estudiantes.

\section{Materiales y métodos}

La investigación se desarrolló de forma comparativa siendo de tipo cuasi experimental, se comparó el desempeño académico en la asignatura de matemáticas, específicamente en la Unidad Didáctica: Las Cónicas, abordando los contenidos de circunferencia, parábola, elipse e hipérbole. Este trabajo se lo desarrolló en el primer parcial de primer quimestre. La población con la que se hizo la investigación fueron los estudiantes de Segundo año de Bachillerato en Ciencias durante dos años lectivos diferentes: 2013-2014 y 2014- 2015, siendo el primer grupo quienes no recibieron el apoyo de la pizarra didáctica cartesiana (grupo de control) y el segundo grupo aquel que trabajo con este nuevo instrumento didáctico en la clase de Matemáticas (grupo experimental), los parámetros académicos que tiene la Unidad Educativa ITSUP son los mismos que proporcionó en estos dos periodos el sistema nacional de evaluación. Luego del proceso de tabulación de datos se realizó el análisis y procesamiento de la información que permitió conocer la influencia de la Pizarra Didáctica Cartesiana en el desempeño académico de los estudiantes en la asignatura de Matemáticas.

\section{Resultados}

En el año lectivos 2013 - 2014 se trabajó con un grupo de 22 estudiantes y en el periodo 20142015 con 20 alumnos, los parámetros que se aplicaron fueron: Tareas Académicas Independientes (TAI), Actividades Individuales en Clase (AIC), Actividades Grupales en Clase (AG), Lecciones (L) y la Evaluación Sumativa (SS), considerando como indicador el promedio final del primer parcial del primer quimestre. El total de la población considerada en la presente investigación fueron 42 estudiantes. Clasificados así: 22 estudiantes grupo control y 20 estudiantes grupo experimental. 
Rev. SINAPSIS, Vol. 5, No 2, Diciembre 2014

La hipótesis planteada fue: "Los estudiantes que aprenden -las cónicas- utilizando la pizarra didáctica cartesiana obtienen mejor desempeño académico en la asignatura de matemáticas". Estadísticamente la hipótesis se la validó con el estimativo " $t$ " por tratarse de muestras cercanas a 20 con un margen de error del 5\%, los resultados obtenidos fueron los siguientes:

\section{Grupo Control} 2013-2014

\begin{tabular}{c|r|r}
\hline Casos & \multicolumn{1}{|c|}{$F$} & $(\mathrm{f}-\mathrm{x})^{2}$ \\
\hline $\mathbf{1}$ & 5,70 & 3,31 \\
\hline $\mathbf{2}$ & 8,00 & 0,23 \\
\hline $\mathbf{3}$ & 7,40 & 0,01 \\
\hline $\mathbf{4}$ & 9,42 & 3,62 \\
\hline $\mathbf{5}$ & 8,90 & 1,91 \\
\hline $\mathbf{6}$ & 7,25 & 0,07 \\
\hline $\mathbf{7}$ & 8,45 & 0,87 \\
\hline $\mathbf{8}$ & 5,60 & 3,68 \\
\hline $\mathbf{9}$ & 8,65 & 1,28 \\
\hline $\mathbf{1 0}$ & 9,85 & 5,44 \\
\hline $\mathbf{1 1}$ & 7,80 & 0,08 \\
\hline $\mathbf{1 2}$ & 6,50 & 1,04 \\
\hline $\mathbf{1 3}$ & 7,26 & 0,07 \\
\hline $\mathbf{1 4}$ & 6,70 & 0,67 \\
\hline $\mathbf{1 5}$ & 8,40 & 0,78 \\
\hline $\mathbf{1 6}$ & 8,30 & 0,61 \\
\hline $\mathbf{1 7}$ & 5,70 & 3,31 \\
\hline $\mathbf{1 8}$ & 5,80 & 2,95 \\
\hline $\mathbf{1 9}$ & 6,86 & 0,43 \\
\hline $\mathbf{2 0}$ & 6,06 & 2,13 \\
\hline $\mathbf{2 1}$ & 7,25 & 0,07 \\
\hline $\mathbf{2 2}$ & 9,56 & 4,17 \\
\hline & $X=7,52$ & $\sum=36,72$ \\
\hline & & \\
\hline
\end{tabular}

\section{Grupo Experimental} 2014-2015

\begin{tabular}{c|c|c}
\hline Casos & $\mathrm{f}$ & $(\mathrm{f}-\mathrm{x})^{2}$ \\
\hline $\mathbf{1}$ & 8,58 & 0,63 \\
\hline $\mathbf{2}$ & 8,07 & 0,08 \\
\hline $\mathbf{3}$ & 10,00 & 4,91 \\
\hline $\mathbf{4}$ & 9,53 & 3,05 \\
\hline $\mathbf{5}$ & 8,78 & 1,00 \\
\hline $\mathbf{6}$ & 8,51 & 0,53 \\
\hline $\mathbf{7}$ & 7,50 & 0,08 \\
\hline $\mathbf{8}$ & 7,46 & 0,10 \\
\hline $\mathbf{9}$ & 7,45 & 0,11 \\
\hline $\mathbf{1 0}$ & 7,36 & 0,18 \\
\hline $\mathbf{1 1}$ & 7,81 & 0,00 \\
\hline $\mathbf{1 2}$ & 9,00 & 1,48 \\
\hline $\mathbf{1 3}$ & 8,29 & 0,26 \\
\hline $\mathbf{1 4}$ & 7,00 & 0,61 \\
\hline $\mathbf{1 5}$ & 10,00 & 4,91 \\
\hline $\mathbf{1 6}$ & 7,31 & 0,22 \\
\hline $\mathbf{1 7}$ & 9,61 & 3,33 \\
\hline $\mathbf{1 8}$ & 7,26 & 0,27 \\
\hline $\mathbf{1 9}$ & 7,29 & 0,24 \\
\hline $\mathbf{2 0}$ & 7,65 & 0,02 \\
\hline & $X=7,78$ & $\sum=22,03$ \\
\hline & & \\
\hline
\end{tabular}

$\mathrm{S}_{2}^{2}=\sqrt{\frac{22.03}{20}}=1.05$ 
Rev. SINAPSIS, Vol. 5, No 2, Diciembre 2014

ISSN 1390 - 7832

Decisión: Los estudiantes que aprenden -las cónicas- utilizando la pizarra didáctica cartesiana obtienen mejor desempeño académico en la asignatura de matemáticas

\section{Discusión}

Sobre pizarras didácticas cartesianas no se ha encontrado trabajo alguno, las investigaciones relativas se las ubica en pizarras electrónicas y con software específicos para la enseñanza de matemáticas; sobre esto resultan interesantes los trabajos realizados en la Universidad Autónoma de Barcelona en la cual se hizo un trabajo de evaluar el uso de esta herramienta didáctica, concluyendo que los resultados del informe europeo sobre las TIC's en la enseñanza hasta el momento demuestra que su uso tiene un impacto positivo sobre los alumnos, mejorando su rendimiento escolar, al mismo tiempo los que tienen mayores dificultades de aprendizaje salen fuertemente beneficiados con este tipo de innovación. (Aragon Educa Co, 2010).

A nivel de Latinoamérica, en la Universidad Nacional de la Plata (Argentina) Rubén A. Pizarro diseño un software educativo, que pretende implementar como herramienta que permita al docente contar con un nuevo recurso didáctico a partir del cual se puedan abordar de manera simple pero con el rigor matemático necesario, los contenidos relacionados con la resolución numérica de ecuaciones no lineales. Especialmente, la visualización gráfica de cómo cada método de resolución va produciendo los resultados de acuerdo a su funcionamiento. Es claro que es imposible lograr esta visualización en una clase tradicional; esto es, a través de la exposición y explicación del tema en el pizarrón. Por medio del aporte de la tecnología a la enseñanza de Cálculo Numérico, se pueden incluir la animación, la dinámica y la interactividad necesaria con el objetivo de facilitar y mejorar la enseñanza de los métodos numéricos como así también su aprendizaje. (Pizarro, 2009).

En Ecuador se encontró un estudio bastante interesante realizado en la Universidad Tecnológica Israel, dicho trabajo fue realizado por el estudiante Byron Patricio Andrade Carreño, bajo el título: "ESTUDIO DEL USO DE LA PIZARRA DIGITAL INTERACTIVA PARA DESARROLLAR LAS COMPETENCIAS DE LOS ALUMNOS EN EL ÁREA DE MATEMÁTICA A NIVEL SECUNDARIO”, dicho trabajo lo realizó en base a encuestas a docentes del nivel secundario en dos establecimientos educativos de la provincia de AzuayEcuador, concretamente en los Colegios FASAYÑAN y NACIONAL CHORDELG, obteniendo 
Rev. SINAPSIS, Vol. 5, No 2, Diciembre 2014

ISSN 1390 - 7832

como resultados que los 11 docentes de matemáticas encuestados coinciden en manifestar que es necesario utilizar la pizarra digital interactiva en el desarrollo de los aprendizajes de los estudiantes, en sus encuestas realizadas los valores porcentuales indican que el $73 \%$ de los docentes utilizan la computadora para sus clases, lo que implica que el $27 \%$ no aplica la tecnología.

También se destaca que de los 11 docentes, 4 tiene pizarras interactivas en sus establecimientos, por lo que el $64 \%$ desconocen de esta herramienta, coincidiendo que no la han utilizado, el $73 \%$ de los docentes indica que si tiene conocimientos para utilizarla mientras que el $27 \%$ no tiene conocimientos para poderla aplicar en sus clases respectivas, lo que requiere una capacitación para poder emplear la tecnología.

Complementario al trabajo realizado por Andrade, realizó encuestas a una muestra de 161 estudiantes de los colegios anteriormente citados y sus resultados se mostraron de la siguiente manera: el 65\% de la muestra indica que conocen lo que es una pizarra didáctica digital, el 35\% desconoce. El 88\% le gustaría conocer el funcionamiento de la pizarra digital interactiva PDI, al $12 \%$ no le gustaría. En exactamente los mismos porcentajes indican que le gustaría que la empleen en las clases de matemáticas, el 100\% indica que si se utilizan nuevas tecnologías hará que el rendimiento académico en matemáticas mejore (Andrade, 2012)

Otro trabajo interesante es el realizado en la Universidad Central del Ecuador, se trata de un proyecto de investigación previo a la obtención de grado de Licenciatura en Ciencias de la Educación, con mención: Informática Aplicada a la Educación, bajo el tema: “TIC'S EN EL RENDIMIENTO ESCOLAR EN LA ASIGNATURA DE MATEMÁTICA EN ESTUDIANTES DE SEGUNDO AÑO DEL B. G. U. DEL COLEGIO NACIONAL SANTIAGO DE GUAYAQUIL, DEL D. M. Q. PERIODO LECTIVO 2011 - 2012”, elaborado por Yury Alex Orozco Muñoz quien aplicó en su instrumento de investigación de campo, la encuesta a los estudiante del mencionado colegio, las mismas que indicaron lo siguiente:

El 71,1\% de los estudiantes encuestados, consideran que las al utilizar las TIC facilitan un mejor aprendizaje, un 24,4\% considera que a menudo y el 4,4\% responde rara vez. Se puede interpretar que los estudiantes consideran que la utilización de las TIC genera aprendizajes significativos. 
Rev. SINAPSIS, Vol. 5, No 2, Diciembre 2014

ISSN $1390-7832$

El $42 \%$ de los estudiantes encuestados, consideran que el profesor de matemática rara vez dispone de un adecuado conocimiento en el uso de las herramientas tecnológicas, un $31 \%$ considera que a menudo, el $25 \%$ responde siempre y el $2 \%$ responde nunca.

Los estudiantes encuestados consideran que, el $47 \%$ de los docentes rara vez utilizan herramientas de tipo tecnológico un $31 \%$ considera que nunca, el $11 \%$ responde a menudo y el $11 \%$ responde que siempre.

De acuerdo a lo determinado por los estudiantes encuestados, el $78 \%$ consideran que siempre que se apliquen herramientas novedosas en la asignatura de matemática se puede desarrollar de mejor manera sus habilidades y un $22 \%$ considera que a menudo.

A los docentes también se le realizó este tipo de investigación de campo, los mismos que dieron como resultados más significativos, los siguientes:

El 75\% de los docentes encuestados, responden que rara vez utilizan en sus clases herramientas de tipo tecnológico y un $25 \%$ responde que si lo hace, los porcentajes obtenidos de los docentes el $75 \%$ consideran que siempre al desarrollar los contenidos de la asignatura de matemática se puede desarrollar las habilidades y destrezas en los estudiantes y un $25 \%$ considera que de vez en cuando.

En iguales proporciones los docentes encuestados, consideran que siempre el uso de la pizarra

digital es una solución para mejorar el rendimiento académico en la asignatura de matemática y el $25 \%$ considera que tal vez, concluyendo que la utilización de herramientas tecnológicas son necesarias para obtener un mejor aprendizaje y desarrollar fácilmente las capacidades cognitivas de los estudiantes (Orozco, 2013)

Como se ha podido demostrar, el aporte que los autores de este esta investigación es significativo ya que no existen rastros de investigaciones concernientes a pizarras cartesianas didácticas.

\section{Conclusiones}

Las herramientas de manipulación concreta didácticamente utilizadas, mejora el desempeño académico de los estudiantes, comprobado por la cuasi experimentación realizada en la 
Rev. SINAPSIS, Vol. 5, No 2, Diciembre 2014

ISSN 1390 - 7832

investigación, requiriendo que el docente reflexione sobre la necesidad de prepararse o de preparar nuevas herramientas digitales o materiales para trabajar contenidos específicos en la asignatura de matemática; la pizarra didáctica tiene esa característica.

La pizarra didáctica cartesiana es sencillamente una herramienta de trabajo para el docente de matemática, fue diseñada pensando en aspectos importantes de las actividades que les llama la atención a los estudiantes, lo cual es la manipulación, sin llegar a digitalizaciones, y como estrategia para hacer más atractivo en aprendizaje de las operaciones cartesianas.

Las herramientas siempre son visuales, es decir que el estudiante se siente más cómodo interactuando que escribiendo o haciendo cálculos, se siente mejor manipulando y haciendo gráficas, pero de manera muy especial en la pizarra didáctica cartesiana ya que le permite sentir con sus propias manos el movimiento correcto que debe realizar, y lo relevante para tomar apuntes y sacar sus propias conceptualizaciones.

\section{Bibliografía}

1. Andrade, B. (07 de 11 de 2012). Estudio del uso de pizarras digitales interactivas para desarrollar las competencias matemáticas. Quito, Pichincha, Ecuador.

2. Aragon Educa Co. (2010). Evaluacion del programa pizarra digital. Universidad Autonoma de Barcelona, 17-20.

3. ARSA. (2012). Aprendizaje de las Matematicas mediante materiales Digitales y Didacticos. Habilidades Digitales para Todos, 6.

4. Carvajal, M. (2000). Educacion Matematica y Didactica de las Matematicas . Educacio Matematica, 1-12.

5. Cornejo, J. (2010). Historia de las Matemáticas. Madrid: Universitaria.

6. Gallego, D., \& Cacheiro, M. L. (2009). La Pizarra Digital Interactiva como recurso docente. Revista Electrónica Teoria de la Educación, 129.

7. Medina, A., \& Francisco, M. (2009). Didactica General. Madrid: Pearson .

8. Medina, A., \& Mata, F. (2009). Didactica General. Madrid : Pearson.

9. Orozco, Y. (06 de 05 de 2013). TIC's en el rendimiento escolar en la asignatura de matemática en los estudiantes de segundo año de BGU. Guayaquil, Guayas, Ecuador.

10. Pere, G., \& Dvoskin, G. (2010). La pizarra digital en el aula de clases. Barcelona: Edebé. 
Rev. SINAPSIS, Vol. 5, No 2, Diciembre 2014

11. Pizarro, R. (18 de 04 de 2009). Las TICs en la enseñanza de las Matematicas.Aplicacion al caso de Metodos numericos. La Plata, Buenos Aires, Argentina.

12. Silva, J. E. (2011). Economía Solidaria. Economix, 12-18.

13. Toledo, P., \& Sanchez, J. (2014). Situación actual de las pizarras digitales interactivas en las aulas de primaria. Educacion a Distancia, 2-3. 